\title{
Oral post-hydration after primary PCI for STEMI
}

\author{
Jonas Dominik Häner, Lorenz Räber \\ Department of Cardiology, Inselspital, Bern University Hospital, University of Bern, Bern, Switzerland \\ Correspondence to: Lorenz Räber, MD, PhD. Professor, interventional cardiologist, Department of Cardiology, INSELSPITAL, Bern University \\ Hospital, University of Bern, 3010 Bern, Switzerland. Email: lorenz.raeber@insel.ch. \\ Comment on: Song F, Sun G, Liu J, et al. The association between post-procedural oral hydration and risk of contrast-induced acute kidney injury \\ among ST-elevation myocardial infarction patients undergoing primary percutaneous coronary intervention. Ann Transl Med 2019;7:321.
}

Submitted Jul 26, 2019. Accepted for publication Aug 07, 2019.

doi: $10.21037 / \mathrm{atm} .2019 .08 .27$

View this article at: http://dx.doi.org/10.21037/atm.2019.08.27

In patients undergoing percutaneous coronary intervention for ST-segment myocardial infarction (STEMI), acute kidney injury (AKI) occurs in $~ 11 \%(\sim 33 \%$ when presenting in cardiogenic shock), as compared to $\sim 3 \%$ in patients with stable angina (1). AKI in patients with acute myocardial infarction is a multifactorial complication of the underlying acute cardiac disease and its treatment (Figure 1, left panel). Hemodynamic changes, arrhythmia and increased sympathetic activation directly reduce renal arterial blood flow. The kidney's unique microvasculature which first supplies the glomeruli via the afferent glomerular arteriole and only subsequently the renal medulla via the efferent arteriole, peritubular network and finally the vasa recta puts the renal medulla at an extraordinary susceptibility for ischemic damage in acutely ill patients. Increased thrombogenicity during acute coronary syndromes and microemboli during cardiac catheterization may also contribute to renal damage. Finally, iodinated contrast dye further deteriorates renal hemodynamics by secondary arteriolar vasoconstriction (after initial endotheliumdependent vasodilation) and importantly causes direct tubular toxicity resulting in acute tubular necrosis, which begins within minutes after exposition and remains toxic over days (2).

Contrast-associated acute kidney injury (CA-AKI) is usually defined as a serum creatinine increase of $0.5 \mathrm{mg} / \mathrm{dL}$ or $25 \%$ from the baseline level and develops in the first 2-5 days after exposition to iodinated contrast media. CA-AKI correlates with development of chronic kidney disease and increased risk of death, stroke and myocardial infarction (3). The corner stone of CA-AKI-prevention is the expansion of intravascular volume and increase in renal filtration rate (Figure 1, right panel). The recent 2-by-2 factorial PRESERVE-trial (4) showed no additional benefit of intravenous administration of sodium bicarbonate over intravenous sodium chloride in preventing death, need for dialysis or persistent $\geq 50 \%$ increase in serum creatinine at 90 days in patients undergoing elective angiography. Furthermore, no additional benefit was found for concomitant oral acetylcysteine intake over placebo. Consequently, the use of intravenous isotonic sodium chloride remains the gold standard preventive approach $(2,5)$.

Oral hydration is non-invasive, has low costs and can be continued at home. In a network meta-analysis including 1,754 participants Zhang et al. found oral hydration to be non-inferior to intravenous hydration for the prevention of CA-AKI, but emergency patients were excluded (6).

In this issue of the Annals of Transitional Medicine Song et al. (7) present a pre-specified observational study assessing the effect of adequate $(\mathrm{N}=280)$ versus inadequate $(\mathrm{N}=28)$ oral hydration following primary PCI on the development of contrast-induced AKI and in-hospital outcomes in a cohort of 308 STEMI-patients. AKI was defined as a $25 \%$ or $0.5 \mathrm{mg} / \mathrm{dL}$ increase in serum creatinine from baseline during the first 48-72 hours post-procedure. Adequate oral post-hydration was assumed when oral fluid intake was $>12 \mathrm{~mL} / \mathrm{kg}$ body weight within the first 24 hours after the intervention. The authors found adequate oral post-hydration to be associated with a significantly lower incidence of contrast-induced AKI (22\% vs. $54 \%$, $\mathrm{P}=0.0002)$ and this strategy emerged as protective factor 


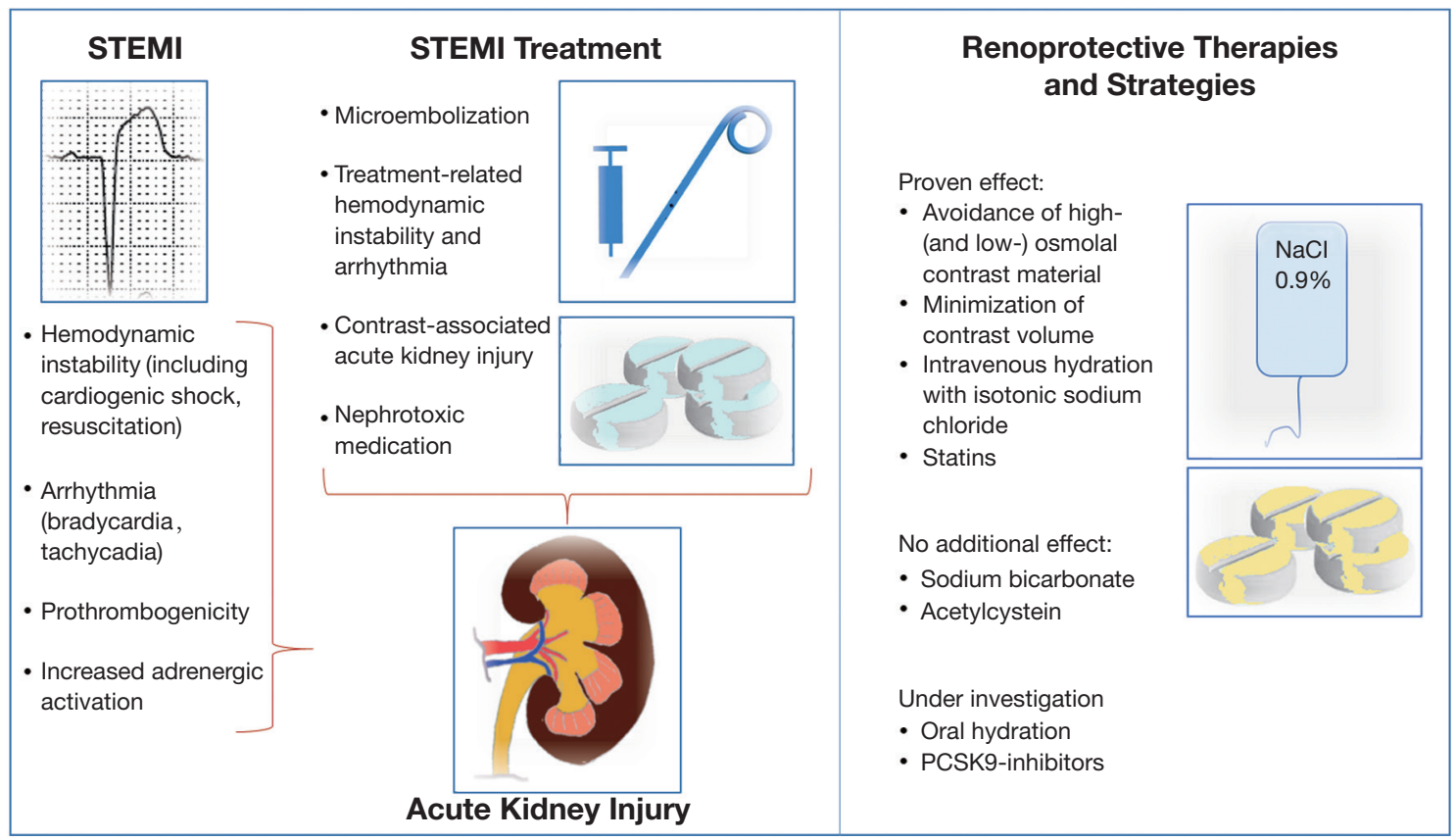

Figure 1 Disease- and treatment-related factors for acute kidney injury in STEMI-patients and renoprotective strategies. Left panel: numerous factors may initiate or aggravate acute renal dysfunction in patients presenting with and treated for STEMI. Mainly hemodynamic instability and arrhythmia due to the acute cardiac disease directly impact renal perfusion. Treatment-related factors, including catheterinduced microembolization and iodinated contrast material further contribute to decreased renal oxygenation or exhibit direct toxic potential to cause renal damage. Right panel: Renoprotection is mainly based on the minimization of contrast material used and intravascular volume expansion using isotonic sodium chloride. STEMI, ST-segment elevation myocardial infarction.

after multivariate adjustments (OR $=0.349,95 \%$ CI: $0.147-$ $0.828, \mathrm{P}=0.0170$ ).

The results of the present study must be interpreted carefully in the light of its major limitations. First, the primary endpoint of AKI was assessed between 48-72 hours postprocedure, which may be too early to detect all cases, taking into account that CA-AKI develops over up to seven days. Second, the authors do not report on the number of patients excluded from the study during the enrolment phase, which in the light of several exclusion criteria (including "missing postoperative oral hydration volume records" and "missing preoperative or postoperative creatinine data") may have been a large number. This may substantially bias the study results. Third, the sample size was rather small, especially the group with inadequate oral post-hydration, which only comprised $10 \%$ of patients ( $\mathrm{N}=28$ ). Fourth, the use of highosmolality contrast agents was excluded but low-osmolal contrast material was allowed. Current guidelines, however, recommend to preferably use iso-osmolal contrast material. Finally and most importantly, although the authors adjusted for several confounders, it is likely that major confounders were not taken into account. The substantial difference in in-hospital survival between the groups (98\% in the adequate oral post-hydration group $v s .61 \%$ in the inadequate oral post-hydration group; and significant differences in stroke and acute heart failure) points towards a massively increased risk of patients allocated to the latter group, not sufficiently addressed and explorated in the baseline characteristics. Infarct size (as for example determined by the peak creatine kinase), mechanical complications, the use of intraaortic balloon pump (in 2012-2013), extracorporeal membrane oxygenation or percutaneous left ventricular assist devices, and the use vasoactive or inotropic medication are neither reported nor included in the multivariate analysis. Congestive heart failure was more frequent in the inadequate oral posthydration group, which represents a Killip II or III class, which is per se associated with worse clinical outcome. Along the same line, the more frequent use of diuretics, the higher Mehran score, the higher age, lower hemoglobin and lower creatinine clearance all indicate worse prognosis for patients in this group. It appears, that patient who did 
not ingest an adequate amount of fluids within the first 24 hours after PCI for STEMI were either hemodynamically unstable or intubated and therefore simply incapable of drinking, or were already volume overloaded (congestive heart failure) rendering adequate oral hydration impossible. As a conclusion, inadequate oral post-hydration served as a risk marker for renal failure and adverse outcomes rather than a causative factor. In this context, the authors conclusion that oral hydration has a practical value in daily life, may count for uncomplicated STEMI patients, but certainly not for the critically ill ones.

Oral pre- and post-hydration may be a comfortable and cheap alternative (or adjunct) to intravenous hydration for clinically stable or stabilized STEMI patients undergoing coronary angiography and intervention. A randomized investigation could address this question but clearly exclude critically ill patients.

\section{Acknowledgments}

None.

\section{Footnote}

Conflicts of Interest: L Räber received research grants to the institution by Abbott Vascular, Biotronik, Boston Scientific, Heartflow, Sanofi and Regeneron and speaker honoraria by Abbott Vascular, Amgen, Astra Zeneca, Biotronik, CSL Behring and Sanofi and Vifor. JH has no conflicts of interest to declare.

Ethical Statement: The authors are accountable for all aspects of the work in ensuring that questions related

Cite this article as: Häner JD, Räber L. Oral post-hydration after primary PCI for STEMI. Ann Transl Med 2019;7(18):425. doi: $10.21037 /$ atm.2019.08.27 to the accuracy or integrity of any part of the work are appropriately investigated and resolved.

\section{References}

1. Tsai TT, Patel UD, Chang TI, et al. Contemporary incidence, predictors, and outcomes of acute kidney injury in patients undergoing percutaneous coronary interventions: Insights from the NCDR cath-PCI registry. JACC Cardiovasc Interv 2014;7:1-9.

2. McCullough PA, Choi JP, Feghali GA, et al. ContrastInduced Acute Kidney Injury. J Am Coll Cardiol 2016;68:1465-73.

3. Bahrainwala JZ, Leonberg-Yoo AK, Rudnick MR. Use of Radiocontrast Agents in CKD and ESRD. Semin Dial 2017;30:290-304.

4. Weisbord SD, Gallagher M, Jneid H, et al. Outcomes after Angiography with Sodium Bicarbonate and Acetylcysteine. N Engl J Med 2018;378:603-14.

5. Mehran R, Dangas GD, Weisbord SD. ContrastAssociated Acute Kidney Injury. N Engl J Med 2019;380:2146-55.

6. Zhang W, Zhang J, Yang B, et al. Effectiveness of oral hydration in preventing contrast-induced acute kidney injury in patients undergoing coronary angiography or intervention: a pairwise and network meta-analysis. Coron Artery Dis 2018;29:286-93.

7. Song F, Sun G, Liu J, et al. The association between postprocedural oral hydration and risk of contrast-induced acute kidney injury among ST-elevation myocardial infarction patients undergoing primary percutaneous coronary intervention. Ann Transl Med 2019;7:321. 